\title{
$\alpha 4 \beta 1$ integrin and 190-kDa CD44v constitute a cell surface docking complex for gelatinase B/MMP-9 in chronic leukemic but not in normal B cells
}

\author{
Javier Redondo-Muñoz, ${ }^{1}$ Estefanía Ugarte-Berzal, ${ }^{1}$ José A. García-Marco, ${ }^{2}$ Mercedes Hernández del Cerro, ${ }^{1}$ \\ Philippe E. Van den Steen, ${ }^{3}$ Ghislain Opdenakker, ${ }^{3}$ María José Terol, ${ }^{4}$ and Angeles García-Pardo ${ }^{1}$ \\ ${ }^{1}$ Departamento de Fisiopatología Celular y Molecular, Centro de Investigaciones Biológicas, Consejo Superior de Investigaciones Científicas, Madrid, Spain; \\ ${ }^{2}$ Servicio de Hematología, Hospital Puerta de Hierro, Madrid, Spain; ${ }^{2}$ Rega Institute for Medical Research, University of Leuven, Leuven, Belgium; and ${ }^{4}$ Servicio \\ de Hematología y Medicina Oncológica, Hospital Clínico, Valencia, Spain
}

\begin{abstract}
As B-cell chronic lymphocytic leukemia (B-CLL) progresses, malignant cells extravasate and infiltrate lymphoid tissues. Several molecules, including gelatinase B/MMP-9, contribute to these processes. Although mainly a secreted protease, some MMP-9 is present at the $B-C L L$ cell surface and the function, mode of anchoring, and interactions of this MMP-9 are unknown. Here we show that anti-MMP-9 antibodies immunoprecipitated a 190-kDa CD44v isoform and $\alpha 4 \beta 1$ integrin from $B-C L L$ cells, but not
\end{abstract}

from normal B cells. Function-blocking antibodies to $\alpha \mathbf{4} \beta 1$ or CD44, or transfection with specific siRNAs, decreased cell-associated proMMP-9 and increased the secreted form. B-CLL cells attached to and bound proMMP-9 and active MMP-9, and this was inhibited by blocking the expression or function of $\alpha 4 \beta 1$ or CD44. The MMP-9 hemopexin domain was critical in these interactions. $\alpha 4 \beta 1$ and 190-kDa CD44v (but not CD44H) formed a complex at the cell surface, since they both coimmunoprecipitated with anti- $\alpha 4$, anti- $\beta 1$, or anti-CD44 antibodies. Immunofluorescence analyses confirmed that $\alpha 4 \beta 1$ and CD44v colocalized with MMP-9. Binding of proMMP-9 inhibited B-CLL cell migration, and this required MMP-9 proteolytic activity. Thus, we have identified $\alpha 4 \beta 1$ and CD44v as a novel proMMP-9 cell surface docking complex and show that cellassociated MMP-9 may regulate $B-C L L$ cell migration and arrest. (Blood. 2008; 112:169-178)

\section{Introduction}

B-cell chronic lymphocytic leukemia (B-CLL) is characterized by the accumulation of $\mathrm{CD}^{+} \mathrm{B}$ lymphocytes in the peripheral blood. ${ }^{1,2}$ As the disease progresses, these cells infiltrate the bone marrow and secondary lymphoid tissue, resulting in poor prognosis. ${ }^{1,2}$ Several molecules that participate in B-CLL cell migration and invasion have been identified. These include the CCL21, CCL19, and CXCL12 chemokines, ${ }^{3,4}$ the vascular endothelial growth factor (VEGF), ${ }^{5}$ and the $\alpha \mathrm{L} \beta 2$ and $\alpha 4 \beta 1$ integrins. ${ }^{5,6}$

Other molecules known to play a role in cell migration are matrix metalloproteinases (MMPs). ${ }^{7-9} \mathrm{~B}-\mathrm{CLL}$ cells produce and secrete progelatinase-B/proMMP-9 $(92 \mathrm{kDa}),{ }^{10}$ and elevated intracellular levels of this MMP correlate with advanced stage and poor patient survival. ${ }^{11}$ We and others have shown that MMP-9 plays an important role in B-CLL transendothelial migration and invasion through basement membranes. ${ }^{6,11} \mathrm{We}$ also recently showed that the constitutive levels of proMMP-9 in B-CLL cells are significantly higher than in normal B cells, and that secretion of proMMP-9 by B-CLL cells is up-regulated by $\alpha 4 \beta 1$ integrin, CXCR4, or CCR7 (the receptors for CXCL 12 and CCL19/CCL21, respectively) engagement. ${ }^{6,12}$ Besides up-regulating proMMP-9 levels, $\alpha 4 \beta 1$ integrin ligation also induces the formation of podosomes in B-CLL cells and the localization of proteolytically active MMP-9 to these invasive structures. ${ }^{6}$

Although mainly present as a secreted soluble form, proMMP-9 has also been detected at the B-CLL cell surface by flow cytometry and cell biotinylation analyses. ${ }^{11}$ Indeed, we recently showed that MMP-9 is present at the B-CLL cell membrane fraction in apparently both the proform and the active form. ${ }^{6}$ Since MMP-9 does not have a transmembrane region, its association to the cell surface requires binding to cell membrane proteins. ${ }^{13}$ In other cell types, these proteins include CD44 (murine carcinoma and human melanoma cells), ${ }^{14,15} \alpha \mathrm{L} \beta 2$ and $\alpha \mathrm{M} \beta 2$ integrins (neutrophils), ${ }^{16}$ the $\alpha 2$ chain of collagen IV (breast epithelial cells), ${ }^{17}$ and the membrane form of the DNA repair protein $\mathrm{Ku}$ (monocytic cells). ${ }^{18}$ Targeting MMP-9 to the cell surface is thought to localize and enhance its proteolytic activity at the pericellular space, and this has been demonstrated upon MMP-9 binding to CD44. ${ }^{15,19}$ In contrast, MMP-9 interaction with RECK (reversion-inducing-cysteine-rich protein with Kazal motifs) apparently inhibits the enzyme, ${ }^{20}$ and binding to LRP (low-density lipoprotein receptor-related protein) leads to MMP-9 internalization. ${ }^{21}$ These previous findings indicate that localization of MMP-9 at the cell surface is complex and appears to regulate multiple aspects of this enzyme. ${ }^{13}$

The function and molecular associations of MMP-9 at the B-CLL cell surface are not known. These are important issues to be clarified since MMP-9 may play a crucial role in the progression of B-CLL. In the present study, we show that MMP-9 (the pro and active forms) specifically binds $\alpha 4 \beta 1$ integrin and a high-molecularweight variant of CD44 at the B-CLL cell surface. These 2 proteins thus constitute a novel membrane docking complex for MMP-9 in
Submitted August 24, 2007; accepted March 5, 2008. Prepublished online as Blood First Edition paper, March 7, 2008; DOI 10.1182/blood-2007-08-109249.

An Inside Blood analysis of this article appears at the front of this issue.

The online version of this article contains a data supplement.
The publication costs of this article were defrayed in part by page charge payment. Therefore, and solely to indicate this fact, this article is hereby marked "advertisement" in accordance with 18 USC section 1734. 
Table 1. Clinical characteristics of B-CLL patients

\begin{tabular}{lcccc}
\hline Patient & Sex/age, $y$ & Stage $^{*}$ & CD38/ZAP-70+ & Ig status \\
\hline 1 & $\mathrm{M} / 59$ & $\mathrm{~B} / \mathrm{II}$ & $+/+$ & $\mathrm{ND}$ \\
2 & $\mathrm{M} / 65$ & $\mathrm{~B} / \mathrm{II}$ & $+/-$ & $\mathrm{ND}$ \\
3 & $\mathrm{~F} / 84$ & $\mathrm{~A} / \mathrm{I}$ & $+/+$ & $\mathrm{ND}$ \\
4 & $\mathrm{~F} / 69$ & $\mathrm{~B} / \mathrm{II}$ & $+/-$ & $\mathrm{ND}$ \\
5 & $\mathrm{M} / 62$ & $\mathrm{~B} / \mathrm{II}$ & $+/+$ & $\mathrm{ND}$ \\
6 & $\mathrm{M} / 80$ & $\mathrm{~A} / 0$ & $-/-$ & Mutated \\
7 & $\mathrm{M} / 62$ & $\mathrm{~A} / 0$ & $-/-$ & Mutated \\
8 & $\mathrm{M} / 78$ & $\mathrm{~A} / 0$ & $+/+$ & Unmutated \\
\hline
\end{tabular}

No patient received therapy.

ND indicates not determined.

${ }^{*}$ According to Binet et $\mathrm{al}^{22}$ and Rai et $\mathrm{al}^{23}$ criteria.

tThe coexpression of CD38 and ZAP-70 is now determined routinely in all B-CLL samples as this analysis apparently has prognostic value. ${ }^{24,25}$

malignant cells. Moreover, we show that cell-bound MMP-9 may regulate B-CLL cell migration and arrest.

\section{Methods}

\section{Patients and cells}

Peripheral blood samples were collected after informed consent was obtained in accordance with the Declaration of Helsinki from 8 B-CLL patients (Table 1) and 2 mantle-cell lymphoma patients. $\mathrm{CD}^{+} \mathrm{B}$ lymphocytes were purified by Ficoll-Hypaque (Nycomed, Oslo, Norway) centrifugation and negative selection with anti-CD3-conjugated Dynabeads (Dynal Biotech ASA, Oslo, Norway). The resulting B-cell population was more than $95 \% \mathrm{CD} 19^{+}$and more than $90 \% \mathrm{CD}^{+}\left(>70 \% \mathrm{CD}^{+}\right.$for mantle B cells), as determined by flow cytometry (Document S1, available on the Blood website; see the Supplemental Materials link at the top of the online article). Tonsillar B lymphocytes were obtained from 2 adult individuals undergoing routine tonsillectomy and purified to more than $95 \% \mathrm{CD} 19^{+}$ and more than $75 \% \mathrm{CD}^{+}$with anti-CD19- and anti-CD5-conjugated Dynabeads. B lymphocytes from 3 healthy donors (PB-BLs) were purified from buffy coat cells by Ficoll-Hypaque centrifugation and anti-CD19- conjugated Dynabeads, resulting in more than $92 \% \mathrm{CD} 19^{+}$cells. The Epstein-Barr virus (EBV)-transformed HUT112 and BRO168 B-cell lines, established from normal B lymphocytes, have been previously reported. ${ }^{6}$ Human umbilical vein endothelial cells (HUVECs) were cultured as reported. ${ }^{6}$

Approval was obtained from the Consejo Superior de Investigaciones Científicas Bioethics Review Board for these studies. Additional materials and methods are available in Document S1.

\section{Results}

\section{$\alpha 4 \beta 1$ Integrin and a high-molecular-weight CD44 isoform coimmunoprecipitate with proMMP-9 in B-CLL cells}

We recently showed that $\alpha 4 \beta 1$ integrin regulates proMMP-9 secretion and localization in podosomes in B-CLL cells. ${ }^{6} \mathrm{~A}$ fraction of the secreted proMMP-9 may bind to the cell surface as MMP-9 is consistently detected at the B-CLL cell membrane. ${ }^{6,11} \mathrm{We}$ therefore investigated whether $\alpha 4 \beta 1$ integrin could interact with surface-associated MMP-9. B-CLL cells from 3 different patients were cultured for 24 hours and lysed, and lysates incubated with an anti-MMP-9 pAb bound to protein A-Sepharose. The presence of MMP-9 in the immunoprecipitates was confirmed by gelatin zymography. This analysis showed a major 92-kDa band, corresponding to proMMP-9 and a minor band of $120 \mathrm{kDa}$, likely representing the proMMP-9/lipocalin complex previously described in B-CLL cells ${ }^{11}$ (shown in Figure 1A for 2 representative patients). Further analysis of these immunoprecipitates by Western blotting revealed the presence of 2 bands of $140 \mathrm{kDa}$ and $120 \mathrm{kDa}$, which corresponded to the $\alpha 4$ and $\beta 1$ integrin subunits, respectively (Figure 1B). Identical results were obtained for patient 3 (not shown). The $\alpha 4$ (Figure 1B) or $\beta 1$ (not shown) bands were not present in control immunoprecipitates in which rabbit IgG was incubated with the cell lysates. Although B-CLL cells also express $\alpha \mathrm{L} \beta 2$ integrin and, in some cases, $\alpha 3 \beta 1$, these integrins did not coimmunoprecipitate with proMMP-9 (results not shown).
A

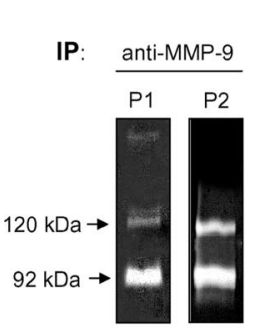

D

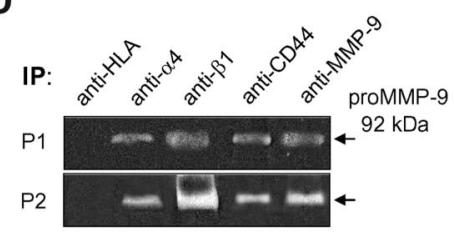

B

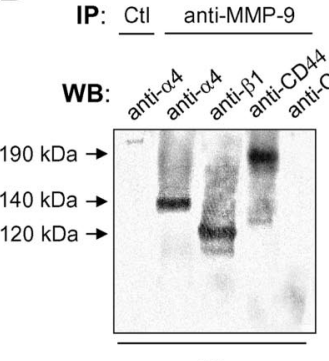

P1 $\underline{\text { Ctl }}$ anti-MMP-9

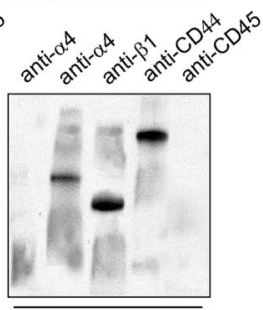

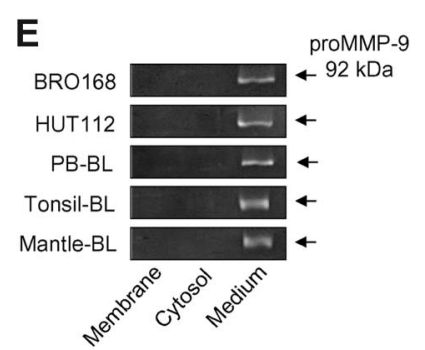

C
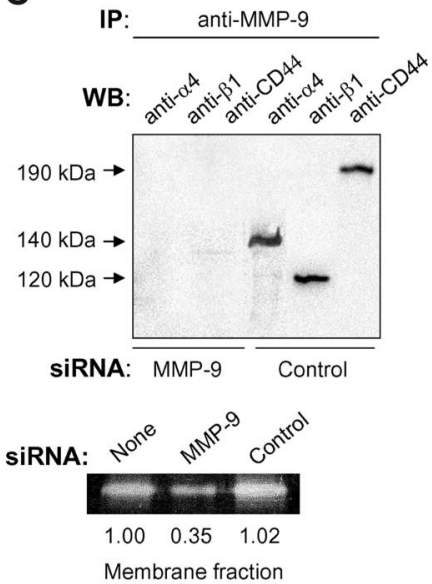

Figure 1. proMMP-9 interacts with $\alpha \mathbf{4 \beta 1}$ integrin and a high-molecular-weight CD44 isoform in B-CLL cells. Lysates from $2 \times 10^{7} \mathrm{~B}-\mathrm{CLL}$ cells cultured for 24 hours in RPMI/0.1\% FCS were immunoprecipitated with anti-MMP-9 pAbs or with rabbit IgG (Ctl), and analyzed by gelatin zymography (A) and Western blotting (B). The results from 2 patients of the 3 analyzed with identical results are shown. (C) Lysates from $2 \times 10^{7}$ B-CLL cells transfected with MMP-9 or control siRNA were immunoprecipitated with anti-MMP-9 pAbs and analyzed by Western blotting. The efficiency of the siRNA transfection was confirmed by gelatin zymography. (D) B-CLL cell lysates were immunoprecipitated with the indicated Abs and analyzed by gelatin zymography. Anti-HLA and anti-MMP-9 Abs were used as negative and positive controls, respectively. (E) Gelatin zymographic analysis of the cell fractions and conditioned media of the indicated B-cell types, after 24-hour cell culture. 
Because CD44 is known to bind MMP-9 in several cell types, ${ }^{14,15,19}$ we also analyzed the presence of CD44 in the proMMP-9 immunoprecipitates of B-CLL cells. As shown in Figure 1B, the anti-CD44 Ab recognized a single band of $190 \mathrm{kDa}$, probably corresponding to a high-molecular-weight CD44 isoform (known as CD44v), while the standard 85/95-kDa CD44 (known as $\mathrm{CD} 44 \mathrm{H}$ ) was not detected (Figure 1B). Another membrane protein, CD45, was also absent in these immunoprecipitates (Figure 1B), confirming the specificity of the coimmunoprecipitation of proMMP-9 with $\alpha 4 \beta 1$ and $190-k D a$ CD44v. Identical results were obtained when proMMP-9 was immunoprecipitated from lysates of freshly isolated cells, which had not been in culture for 24 hours (Figure S1).

To further establish the specificity of the anti-MMP-9 Ab, we analyzed the lysates of B-CLL cells that had been transfected with either MMP-9 siRNA or a control siRNA. The anti-MMP-9 Ab did not immunoprecipitate the $\alpha 4$ or $\beta 1$ integrin subunits or $190-\mathrm{kDa}$ CD44v from MMP-9 siRNA-transfected cells, while these proteins were clearly visible in proMMP-9 immunoprecipitates from control siRNA-transfected cells (Figure 1C). Gelatin zymographic analysis confirmed that the MMP-9 siRNA reduced the amount of membrane-bound and secreted proMMP-9 by 3-fold, compared with untransfected or control siRNA-transfected cells (shown in Figure 1C for membrane-bound proMMP-9).

To then determine whether Abs to $\alpha 4 \beta 1$ integrin or CD44 pulled down proMMP-9, B-CLL cell lysates were incubated with specific Abs to $\alpha 4, \beta 1$, or $\mathrm{CD} 44$, and the immunoprecipitates analyzed by gelatin zymography. As shown in Figure 1D, all 3 Abs pulled down proMMP-9, while anti-HLA Abs did not. The amount of proMMP-9 in these immunoprecipitates was similar to that pulled down by anti-MMP-9 Abs, used as positive control (Figure 1D).

To establish if the observed presence and interactions of surface-bound proMMP-9 also occurred in non-B-CLL B cells, normal peripheral blood B lymphocytes, BRO168 and HUT112 cells, $\mathrm{CD}^{+}$tonsillar B cells, and $\mathrm{CD}^{+}$mantle lymphoma B cells were cultured for 24 hours. The membrane and cytosolic fractions were then separated and analyzed by gelatin zymography. Figure $1 \mathrm{E}$ shows that proMMP-9 was clearly present in the conditioned media of these cells, but absent in their cellular fractions.

\section{Blocking $\alpha 4 \beta 1$ or CD44 function with antibodies or peptides significantly reduces the levels of B-CLL cell surface-associated proMMP-9}

To determine whether $\alpha 4 \beta 1$ and $190-\mathrm{kDa} C D 44 \mathrm{v}$ serve as surface receptors for proMMP-9, we first studied the effect of blocking $\alpha 4 \beta 1$ function on cell surface-bound proMMP-9. Equal numbers of cells from 3 different patients were incubated with either the HP2/1 mAb or the CS1 peptide, 2 well-known inhibitors of $\alpha 4 \beta 1$ function $^{26-28}$; after 24 hours, the membrane and cytosolic cellular fractions were separated and analyzed by gelatin zymography. In agreement with our previous results, ${ }^{6}$ no proMMP-9 was detected in the cytosolic fraction (not shown). The levels of membraneassociated proMMP-9 were significantly reduced $(P \leq .01)$ after cell incubation with HP2/1 or CS1 (Figure S2A left panel). In contrast, the HP1/7 mAb, which does not block $\alpha 4 \beta 1$ function ${ }^{26}$ or the control peptide CS3, ${ }^{27,28}$ had no effect (Figure S2A).

To determine whether the reduced expression of membranebound proMMP-9 correlated with increased secreted levels of this MMP, we analyzed the conditioned media of the same 3 samples by gelatin zymography. As shown in Figure S2A (right panel), cell treatment with HP2/1 or CS1 significantly increased $(P \leq .05)$ the amount of secreted proMMP-9, while incubation with HP1/7 or CS3 had no effect.

We next performed similar experiments to study the effect of blocking CD44 function on the amount of surface-bound and secreted proMMP-9. As shown in Figure S2B, incubation of B-CLL cells (2 patients) with anti-CD44 mAb significantly reduced $(P \leq .05)$ membrane-associated proMMP-9 and increased $(P \leq .05)$ secreted proMMP-9. The control anti-HLA mAb had no effect (Figure S2B). None of the inhibitors of $\alpha 4 \beta 1$ or CD44 function affected the levels of MMP-9 mRNA, as confirmed by reverse transcription-polymerase chain reaction (RT-PCR; not shown). These results indicated that both $\alpha 4 \beta 1$ integrin and CD44 were important for maintaining proMMP-9 at the B-CLL cell membrane.

\section{Knocking down the expression of the $\beta 1$ integrin subunit or CD44 in B-CLL cells significantly reduces the amount of surface-bound proMMP-9 and increases the levels of secreted proMMP-9}

To confirm the results obtained by blocking the function of $\alpha 4 \beta 1$ or CD44, we transfected B-CLL cells (3 patients) with 2 different siRNAs for the $\beta 1$ integrin subunit, since $\alpha 4 \beta 1$ is the major integrin in these cells, ${ }^{29,30}$ or for CD44, or with a control siRNA. siRNA transfection had a minor effect on B-CLL cell viability (shown in Figure S3 for 2 representative samples). Western blotting analyses confirmed that both $\beta 1$ siRNAs significantly reduced (average $52 \%$ and $57 \%$ reduction, $P \leq .05$, for siRNA $_{1}$ and siRNA 2 , respectively, with similar results for all 3 samples) the total levels of $\beta 1$, with respect to untransfected or control siRNA-transfected cells (Figure S4A). Likewise, both CD44 siRNAs significantly reduced (average $60 \%, P \leq .01$, and $67 \%$ reduction, $P \leq .001$, for siRNA $_{1}$ and $\mathrm{siRNA}_{2}$, respectively) the levels of CD44H, while 190-kDa CD44v was no longer detectable (Figure S4B).

Surface expression of the $\beta 1$ and $\alpha 4$ integrin subunits was also effectively reduced by both $\beta 1$ siRNAs (shown in Figure $2 A$ for $\beta 1$ siRNA $_{2}$ and for 2 representative samples). This siRNA reduced the MFI average values from 7.8 to 2.2 , and from 22.6 to 3.8 , for the $\beta 1$ and $\alpha 4$ subunits, respectively, representing a $71 \%$ and $80 \%$ reduction on the surface expression of these subunits. The control siRNA had no effect (Figure 2A). All 3 samples analyzed gave similar results. The levels of CD44 on $\beta 1$ siRNA-transfected cells did not change (Figure 2A), indicating that siRNA transfection did not affect the expression of other membrane proteins. Concomitant with the blockage of $\alpha 4 \beta 1$ surface expression, zymographic analyses revealed that proMMP-9 levels were significantly reduced by both $\beta 1$ siRNA 1 and $\operatorname{siRNA}_{2}(P \leq .05$ and $P \leq .01$, respectively) in the cell membrane fraction, while they were not affected by the control siRNA (Figure $2 \mathrm{~B}$ ). In contrast, and as previously observed by inhibiting $\alpha 4 \beta 1$ function, the levels of secreted proMMP-9 were significantly increased $(P \leq .05)$ on $\beta 1$ siRNAtransfected cells (Figure 2B).

CD44 surface expression was also effectively blocked after CD44 siRNA 2 (or siRNA 1 , not shown) transfection, with average MFI values reduced from 19.2 to 2.1 , or $91 \%$ reduction, while the control siRNA had no effect (Figure 2C). The surface expression of the $\alpha 4$ or $\beta 1$ integrin subunits was not affected by transfection with CD44 siRNAs (Figure 2C). Zymographic analysis of the cell membrane fraction showed that both CD44 siRNAs, but not the control siRNA, significantly $(P \leq .01)$ reduced the amount of surface-bound proMMP-9 (Figure 2D). This reduction correlated with a significant $(P \leq .05)$ increase of secreted proMMP-9 (Figure 2D). 
A

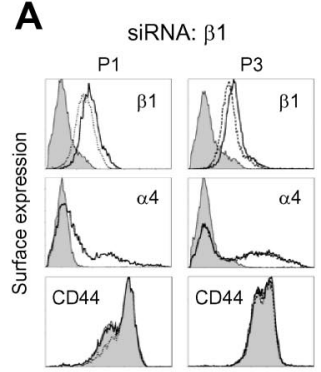

C

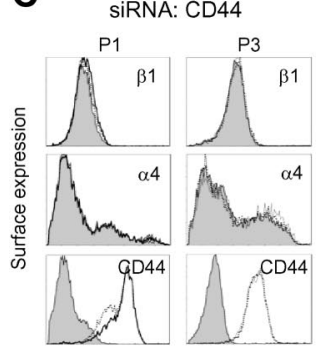

B
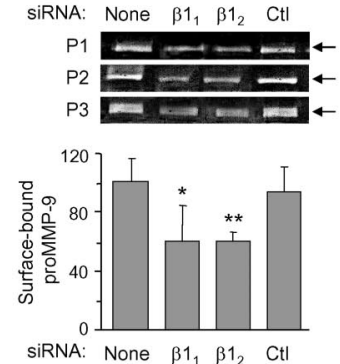

D
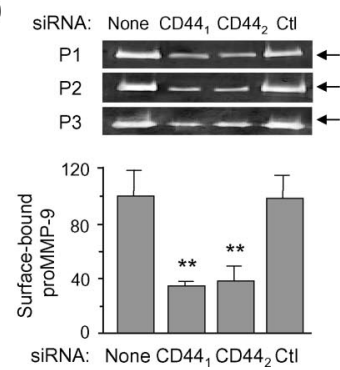

Figure 2. Effect of $\beta 1$ integrin subunit or CD44 gene

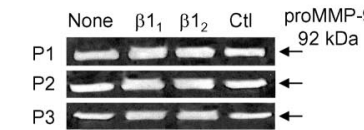
encing on B-CLL cell membrane-bound and secreted proMMP-9. (A) B-CLL cells were transfected with control or $\beta 1$ siRNA and analyzed by flow cytometry. Shaded areas represent $\beta 1$ siRNA-cells; dotted lines, control siRNA cells; and continuous lines, untransfected cells. Representative results from 2 samples of the 3 studied are shown. (B) Gelatin zymographic analysis of proMMP- 9 on the membrane fraction and the conditioned media of untransfected or siRNA-transfected B-CLL cells. $\beta 1_{1}$ and $\beta 1_{2}$ indicate the 2 different $\beta 1$ siRNAs used. Ctl indicates control siRNA. Values represent the average of the 3 samples after normalizing the proMMP-9 levels of untransfected cells to 100. (C) Flow cytometric analysis of B-CLL cells (shown for 2 samples) transfected with control or CD44 siRNA. Line codes are as in panel A. (D) Gelatin zymography of proMMP-9 on the membrane fraction and the conditioned media of untransfected or siRNA-transfected B-CLL cells. CD44 $4_{1}$ and CD44 $4_{2}$ indicate the 2 different CD44 siRNAs used. Values represent the average of all 3 patients after normalizing proMMP-9 levels in untransfected cells to $100 .{ }^{*} P \leq .05 ;{ }^{* \star} P \leq .01$.
To confirm these results, we studied whether surface-bound proMMP-9 levels were restored upon longer B-CLL cell culture, thus allowing for $\alpha 4 \beta 1$ and CD44 re-expression. Figure S5A shows that proMMP-9 levels were nearly fully recovered after 72 hours, in correlation with the surface re-expression of $\alpha 4 \beta 1$ and CD44 (Figure S5B).

\section{B-CLL cells bind soluble proMMP-9 and attach to immobilized proMMP-9 via $\alpha 4 \beta 1$ and CD44; normal B cells do not bind or adhere to proMMP-9}

The preceding results indicated that both $\alpha 4 \beta 1$ integrin and 190-kDa CD44v coimmunoprecipitated with proMMP-9 and were necessary to localize proMMP-9 at the B-CLL cell surface. To further establish that a direct interaction existed between these 2 proteins and proMMP-9, we analyzed the binding of soluble proMMP-9 to B-CLL cells by flow cytometry. These analyses revealed that the constitutive surface expression of proMMP-9 increased 2.4-fold (average of 3 patients with similar results) upon incubating the cells with soluble proMMP-9 (Figure 3A). This indicated that soluble proMMP-9 could bind to the B-CLL cell surface. To determine whether $\alpha 4 \beta 1$ integrin and/or CD44 were involved in this binding, we incubated the cells with the HP2/1 $\mathrm{mAb}$, the $\mathrm{CS} 1$ peptide, or an anti-CD44 $\mathrm{Ab}$, prior to adding proMMP-9. Inhibiting the function of $\alpha 4 \beta 1$ or CD44 significantly $(P \leq .05)$ reduced the expression of surface-bound proMMP-9 to constitutive levels, while the control HP1/7 mAb or the inactive CS3 peptide had no effect (Figure 3A).

To confirm the above results, we performed similar binding experiments using B-CLL cells transfected with control, $\beta 1$, or CD44 siRNAs. Figure 3B shows, for a representative patient, that gene silencing of any of these 2 proteins blocked soluble proMMP-9 binding to B-CLL cells, while the control siRNA had no effect. All 3 patients studied gave identical results, with average MFI values significantly decreasing $(P \leq .05)$ from 3.4 (untransfected or control siRNA-transfected cells) to basal levels (1.3). As a control for the integrity of the membrane after these transfections, HLA expression was similar for all 3 siRNA conditions (Figure 3B) and for untransfected cells (not shown). These results established that both $\alpha 4 \beta 1$ integrin and CD 44 are required for soluble proMMP-9 binding to B-CLL cells. Further analyses of these interactions revealed that proMMP-9 remained bound to the cell surface for the 24 hours of the assay (Figure S6A), that binding was saturable (Figure S6B), and that it did not result in internalization of the $\alpha 4 \beta 1 / \mathrm{CD} 44$ /proMMP-9 complex (Figure S6C).

We then determined if non-B-CLL cells could also bind soluble proMMP-9. In agreement with the immunoprecipitation experiments shown in Figure 1E, BRO168 cells did not express membrane proMMP-9 constitutively (Figure 3C), despite their high expression of $\alpha 4 \beta 1$ integrin (not shown). Moreover, BRO168 cells (or PB-BLs, not shown) were unable to bind soluble proMMP-9, even after activation of the integrin with $\mathrm{Mn}^{2+}$ (Figure 3C). In contrast, $\mathrm{Mn}^{2+}$-treated BRO168 cells efficiently bound soluble VCAM-1, and this binding was inhibited by the CS1 peptide (Figure 3C), indicating that $\alpha 4 \beta 1$ was fully functional in these cells.

We next studied whether proMMP-9 could serve as an anchoring point for B-CLL cells and whether $\alpha 4 \beta 1$ and CD44 mediated this adhesion. B-CLL cells from 3 different patients attached to immobilized proMMP-9 (Figure 3D), and the percentage of adhesion (30\%) was similar to that of cells attached to VCAM-1 (33\%). Each individual sample gave similar results. Blocking the function of $\alpha 4 \beta 1$ integrin with HP2/1 or CS1, abolished cell adhesion to proMMP-9 (and to VCAM-1, not shown). In contrast, the control HP1/7 mAb or the CS3 peptide had no effect. Likewise, cell incubation with the anti-CD44 mAb diminished cell adhesion to proMMP-9 by 53\% (Figure 3D). Moreover, knocking down the expression of $\alpha 4 \beta 1$ or CD44 by specific siRNA transfection significantly $(P \leq .01)$ reduced cell adhesion to proMMP-9 to the basal levels of adhesion to BSA (Figure 3D). In contrast, transfection with a control siRNA had no effect (Figure 3D). These results clearly indicated that proMMP-9 supported adhesion of B-CLL cells and that both $\alpha 4 \beta 1$ integrin and CD44 were required for this adhesion. Interestingly, this dual requirement appeared to be unique for adhesion to proMMP-9, since B-CLL cell adhesion to VCAM-1 was completely blocked by transfection with $\beta 1$ siRNA, but was not affected by transfection with CD44 siRNA (Figure 3D).

To determine whether non-B-CLL B cells attached to immobilized proMMP-9, BRO168 and HUT112 cells, PB-BLs, 


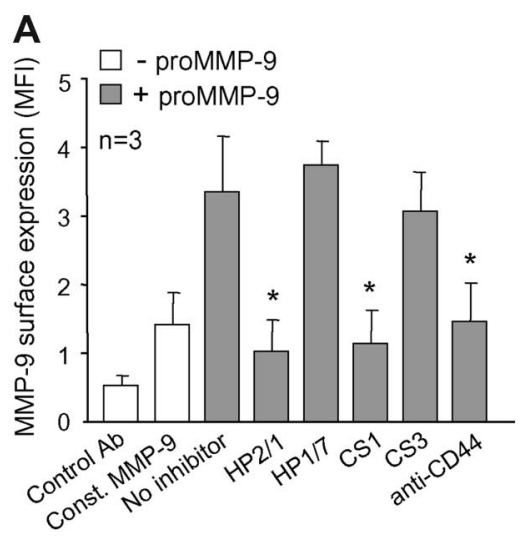

D

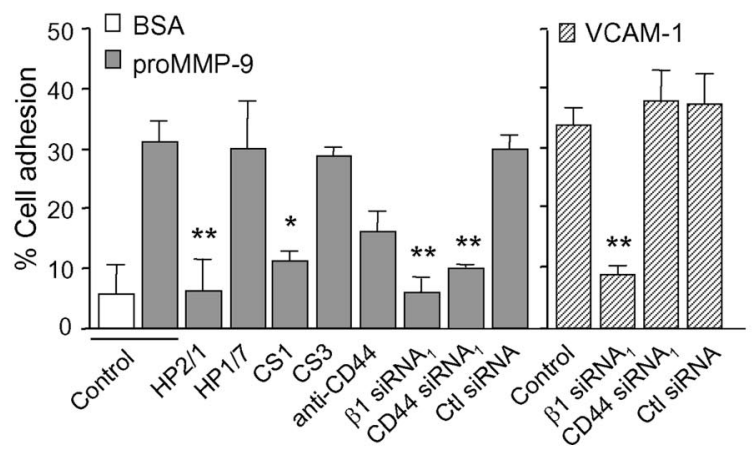

B

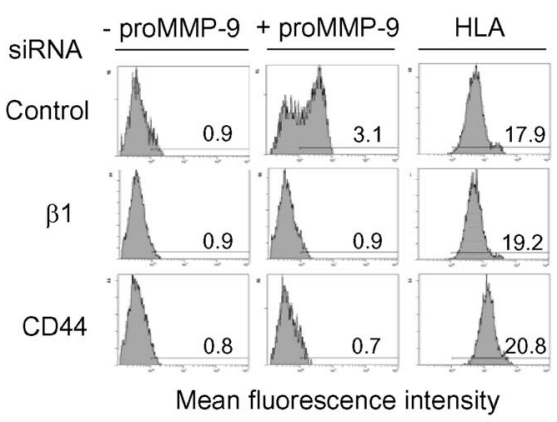

C

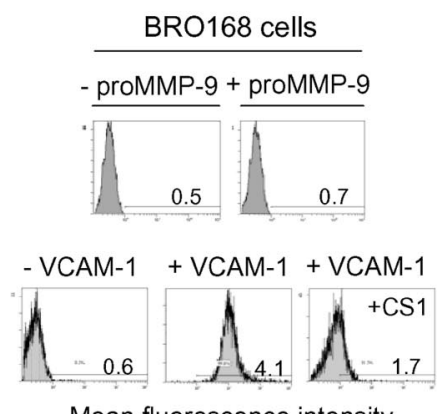

Mean fluorescence intensity

E

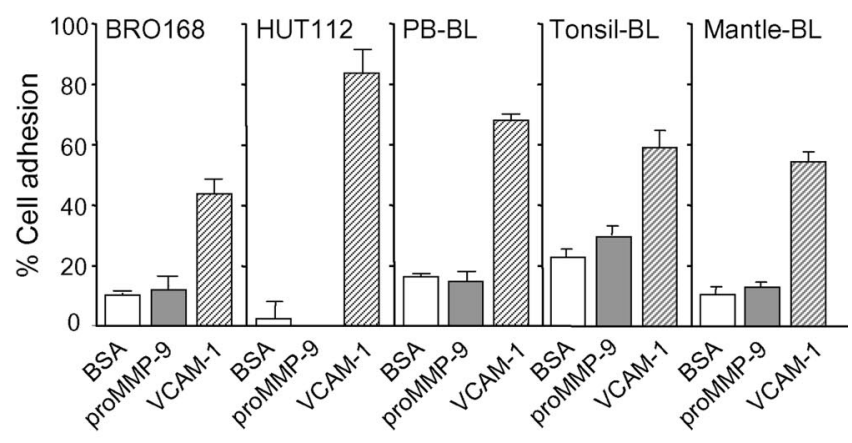

Figure 3. $\alpha 4 \beta 1$ integrin and CD44 mediate soluble proMMP-9 binding to B-CLL cells and adhesion of these cells to immobilized proMMP-9. (A) B-CLL cells, with or without previous incubation with the indicated Abs or peptides, were incubated for 30 minutes with or without proMMP-9 (10 $\mu \mathrm{g} / \mathrm{mL})$ and analyzed by flow cytometry using anti-MMP-9 pAbs. Const MMP-9 indicates constitutive MMP-9. (B) Identical binding experiments were performed with B-CLL cells transfected with the indicated siRNAs. HLA expression was also analyzed as a control for membrane integrity. A representative sample of the 3 studied with identical results is shown. (C) BRO168 cells were incubated with proMMP-9 or VCAM-1 and analyzed by flow cytometry. The binding of VCAM-1 was also measured after cell preincubation with the CS1 peptide. (D) BCECF-AM-labeled B-CLL cells $\left(5 \times 10^{5} ; 3\right.$ patients), with or without previous incubation with the indicated Abs or peptides, or transfected with the indicated siRNAs, were added to wells coated with $10 \mu \mathrm{g} / \mathrm{mL}$ proMMP-9 or VCAM-1. After 90 minutes at $37^{\circ} \mathrm{C}$, attached cells were quantitated using a fluorescence analyzer. Average values, each with duplicate determinations, represent the percentage of the total number of cells added. (E) The indicated BCECF-AM-labeled B cells were added to proMMP-9- or VCAM-1-coated wells and attached cells measured as explained. All determinations were done in triplicate and values represent the average of 3 (BRO168, HUT112, PBL-BL) or 2 (tonsillar and mantle lymphoma $\mathrm{BL}$ ) independent experiments. Bar codes are as in panel $\mathrm{D}$. ${ }^{\star} P \leq .05 ;{ }^{* \star} P \leq .01$.

and $\mathrm{CD}^{+}$tonsillar or mantle lymphoma B cells were used. All these cells attached well to VCAM-1 (Figure 3E), and adhesion was completely inhibited by HP2/1 or CS1 (not shown). In contrast, these cells did not attach to proMMP-9, and this was particularly striking for HUT112 cells, where adhesion to VCAM-1 was nearly $90 \%$ (Figure 3E). Altogether, these results suggested that the ability to use $\alpha 4 \beta 1$ and CD44 as receptors for immobilized or soluble proMMP-9 was a unique characteristic of B-CLL cells.

\section{Further characterization of MMP-9 interaction with B-CLL cells}

To determine which region of proMMP-9 was important for its interaction with B-CLL cells, we used recombinant proteins ${ }^{31}$ representing the full-length proMMP-9 (rproMMP-9fl) or several truncated forms (Figure 4A). B-CLL cells attached to and bound rproMMP-9fl efficiently (Figure 4B,C), and this was inhibited by the HP2/1 and anti-CD44 Abs (83\% and 69\% inhibition, respectively), but not by the HP1/7 Ab (not shown). Removal of the hemopexin domain (rproMMP-9 $\Delta \mathrm{Hem}$ ) or the hemopexin and O-glycosylated (OG) domains (rproMMP-9 $\Delta$ HemOG) significantly reduced $(P \leq .001)$ the ability to support B-CLL cell adhesion (Figure 4B) and the binding to the cell surface $(P \leq .01)$ (Figure 4C) in all 4 patients studied. Removal of the OG domain alone had a minor although significant effect on cell adhesion
$(P \leq .01)$ and soluble binding $(P \leq .05)$. Mutating the catalytic $\mathrm{E}$ residue (rproMMP-9mutE) did not affect the proMMP-9 cell binding property (Figure 4B,C). These results indicated that proMMP-9 interaction with the B-CLL cell surface involves the hemopexin domain and some contribution of the OG domain.

To further extend the physiological relevance of proMMP-9 interaction with B-CLL cells, we studied whether mature/active MMP-9 retained this ability. Treatment of proMMP-9 with APMA completely converted the proform into the reported $83 / 62-\mathrm{kDa}$ MMP-9 active forms ${ }^{8,9}$ (Figure 4D). These active forms mediated cell adhesion and bound to B-CLL cells as efficiently as proMMP-9 (Figure 4E,F). Both events were significantly inhibited in all 3 samples studied by blocking $\alpha 4 \beta 1$ or CD44 with specific Abs or peptides (Figure 4E,F).

\section{The CD44v isoform present in B-CLL cells coimmunoprecipitates with the $\alpha 4$ or $\beta 1$ integrin subunits and is absent in normal or non-CLL $B$ cells}

Since both CD44 and $\alpha 4 \beta 1$ integrin appeared to be receptors for proMMP-9, we studied whether these 2 proteins interacted at the B-CLL surface. Fresh B-CLL cells (2 patients) were lysed and lysates immunoprecipitated with either anti- $\alpha 4$ or anti- $\beta 1$ mAbs, followed by protein G-bound secondary Abs. Western 
A

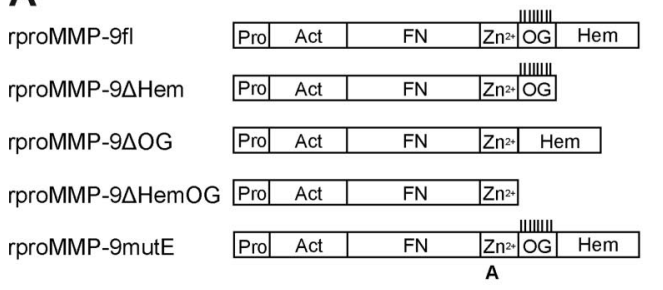

B

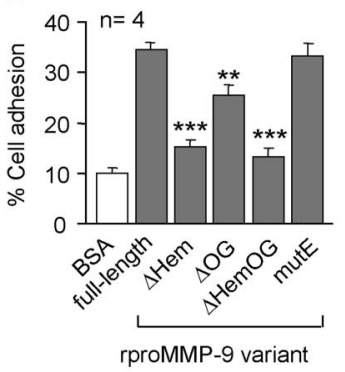

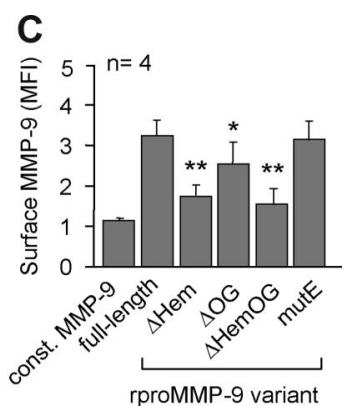

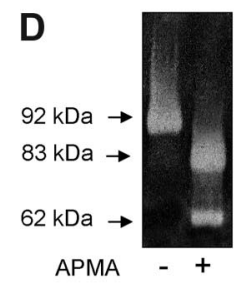

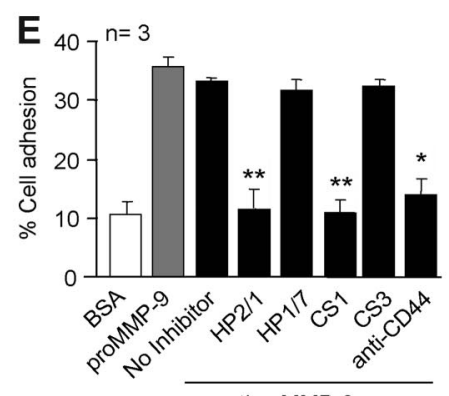

active MMP-9

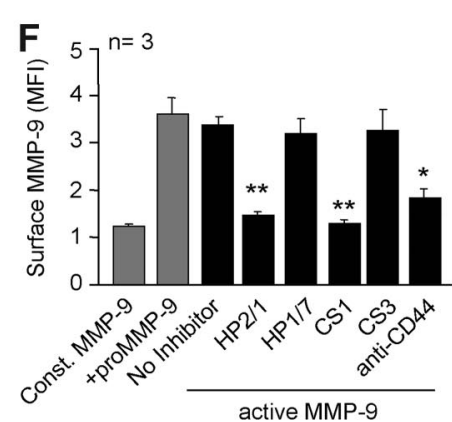

Figure 4. Further characterization of MMP-9 interaction with B-CLL cells. (A) Schematic domain structure of the recombinant full-length (fl) and proMMP-9 variants used (modified from ${ }^{31}$ ). Pro indicates prodomain; Act, active site; FN, gelatin-binding fibronectin-like domain; $\mathrm{Zn}^{2+}$, $\mathrm{Zn}^{2+}$-binding domain; OG, O-glycosylated domain, with vertical lines indicating probable attachment sites for O-linked sugars; and Hem, hemopexin domain. A indicates that the catalytic Glu/E ${ }^{402}$ residue was substituted by alanine. (B) BCECF-AM-labeled B-CLL cells were added to wells coated with the indicated rproMMP-9 variants (110 nM each) and cell adhesion was measured as explained. (C) Binding of soluble rproMMP-9 variants to B-CLL cells, measured by flow cytometry. Values in panels $B$ and $C$ represent the average data from cells of the 4 patients studied. (D) proMMP-9 was treated with $2 \mathrm{mM} \mathrm{APMA}$ for 4 hours at $37^{\circ} \mathrm{C}$ and activation confirmed by gelatin zymography. (E) BCECF-AM-labeled B-CLL cells were added to wells coated with proMMP-9 or active MMP-9 (110 nM each), and cell adhesion was measured as explained. (F) Flow cytometric analysis of the binding of soluble proMMP- 9 or active MMP-9 to B-CLL cells. Values in panels $\mathrm{E}$ and $\mathrm{F}$ represent the averages obtained with cells of 3 patients studied. MFI indicates mean fluorescence intensity. ${ }^{*} \leq .05$; ${ }^{\star *} P \leq .01 ;{ }^{* \star *} P \leq .001$.

blotting analysis of these immunoprecipitates revealed the presence of $190-\mathrm{kDa} C D 44 \mathrm{v}$ and the absence of $95-\mathrm{kDa} C D 44 \mathrm{H}$ (Figure 5A). Moreover, the anti-CD44 mAb immunoprecipitated the $\alpha 4$ and $\beta 1$ integrin subunits (Figure S7). These results suggested that the CD44v/ $\alpha 4 \beta 1$ complex was responsible for the binding of proMMP-9 to the B-CLL cell surface. Because normal or mantle lymphoma B cells did not bind proMMP-9, albeit expressing fully functional $\alpha 4 \beta 1$, we analyzed the presence of the $\mathrm{CD} 44 \mathrm{v}$ isoform on these cells by Western blotting. Figure 5B shows that while the membrane fraction of B-CLL cells contained both the CD44H and CD44v isoforms, all other B cells analyzed contained only CD44H. Further characterization of the CD44v present on the B-CLL samples, with specific Abs to some of the variable exons, indicated that it
A

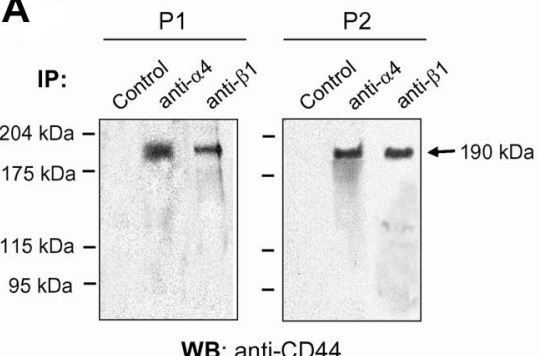

\section{C}

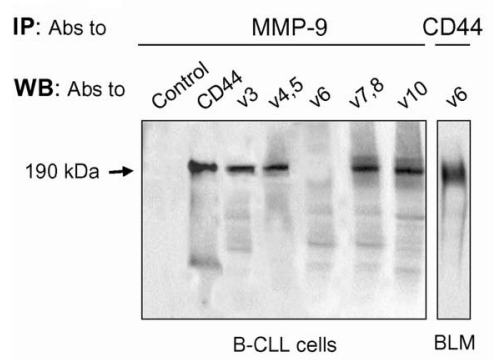

B

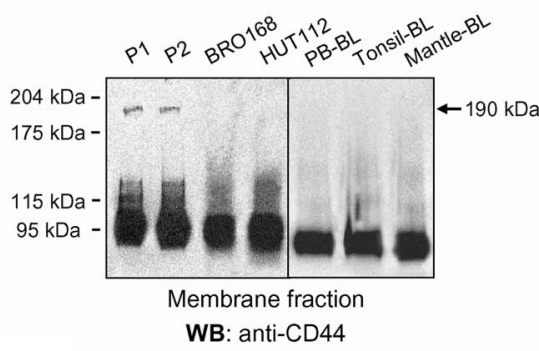

Figure 5. Characterization of CD44 isoforms on CLL and non-CLL B cells. (A) Lysates from $2 \times 10^{7} \mathrm{~B}-\mathrm{CLL}$ cells that had been in RPMI/0.1\% FCS for 24 hours were immunoprecipitated with the indicated Abs (control: antiHLA) and analyzed by Western blotting. (B) The membrane fraction from the same B-CLL samples shown in panel $A$ and from the indicated $B$ cells was analyzed by Western blotting using anti-CD44 Abs. The presence of the $190-\mathrm{kDa}$ CD44v isoform on B-CLL cells is indicated. $A$ vertical line has been inserted to indicate a composition of 2 different gels. (C) The cell lysate from a representative B-CLL sample was immunoprecipitated with antiMMP-9 Abs and analyzed by Western blotting using Abs specific for CD44 variable exons. Lysates from BLM cells were used as control for the anti-v6 mAb. (D) B-CLL cells were incubated with or without proMMP-9 in the presence or absence of the indicated Abs, and binding was analyzed by flow cytometry. Average values are shown. Const MMP-9 indicates constitutive MMP-9. ${ }^{\star} P \leq .05$. 


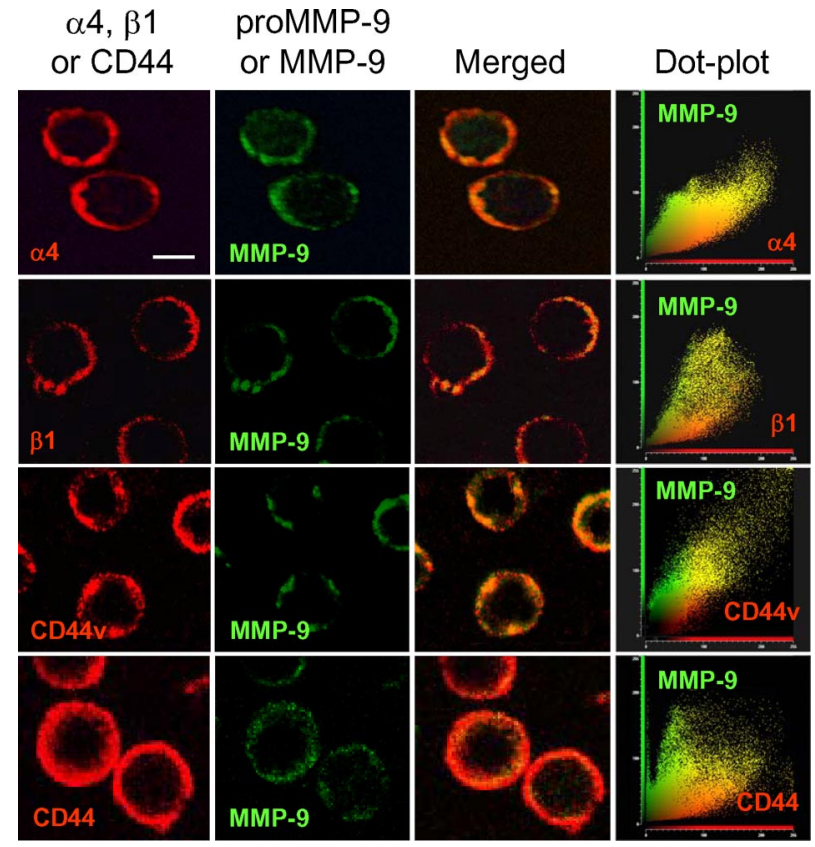

Figure 6. Colocalization of MMP-9 with CD44v and $\alpha 4 \beta 1$ integrin in B-CLL cells. B-CLL cells from a representative sample were added to glass coverslips coated with $5 \mu \mathrm{g} / \mathrm{mL}$ poly-lysine. After 1 hour at $37^{\circ} \mathrm{C}$, cells were fixed and analyzed by confocal microscopy using specific Abs for $\alpha 4, \beta 1$, CD44v7,8, and CD44, followed by Texas-Red-labeled secondary Abs. MMP-9 was detected with specific pAbs and Alexa 488-labeled secondary Abs. Colocalization of MMP-9 with CD $44 \mathrm{v}$ and $\alpha 4 \beta 1$ was further demonstrated using dot-plot analyses. Images were acquired using a confocal scanning inverted AOBS/SP2 microscope (Leica Microsystems, Heidelberg, Germany) with a $63 \times / 1.3$ NA PL-APO glycerol immersion objective. Leica's LCS 15.37 dye-separation software was used for colocalization studies; when necessary, Adobe Photoshop 7.0 (Adobe Systems, San Jose, CA) was used for image processing. Bar represents $4 \mu \mathrm{m}$.

contained v3, v4,5, v7,8, and v10 (shown in Figure 5C for a representative sample). In agreement with a recent report, ${ }^{32} \mathrm{v} 6$ was absent, and we established the functionality of the anti-v6 $\mathrm{Ab}$ on BLM melanoma cells (Figure 5C). These results were confirmed by fluorescence-activated cell sorting (FACS) analysis of the B-CLL and normal B cells (Figure S8). Moreover, the Abs against the CD44 variable exons blocked the binding of soluble proMMP-9 to B-CLL cells in the 3 patients studied (shown in Figure 5D for anti-v7,8 and anti-v10 Abs), confirming the functional implication of these variable exons in the binding of CD44v to proMMP-9.

\section{MMP-9 colocalizes with $\alpha 4 \beta 1$ integrin and CD44v at the B-CLL cell surface, and both receptors are required for MMP-9 cell surface anchoring}

The preceding results indicated that CD44v interacted with $\alpha 4 \beta 1$ and that both proteins served as surface docking receptors for proMMP-9. To confirm that CD44v, $\alpha 4 \beta 1$, and proMMP-9 (or MMP-9 since the Ab did not distinguish between the pro and activated forms) colocalized at the B-CLL cell surface, we performed immunofluorescence analyses using confocal microscopy and focused on cells with higher expression of MMP-9 for easier visualization. As observed in Figure 6 for a representative sample, the $\alpha 4$ or $\beta 1$ subunits, as well as CD44v, colocalized with MMP-9 (pro and/or activated) at the cell periphery, as documented by dot-plot analysis. In contrast, CD44H, which did not coimmunoprecipitate with proMMP-9 (Figure 1B) or $\alpha 4 \beta 1$ (Figure 5A), did not colocalize with MMP-9 (Figure 6), confirming the specific interaction of this MMP with CD44v.
We also analyzed by immunofluorescence the expression of MMP-9 on B-CLL cells transfected with CD44, $\beta 1$, or control siRNAs. Figure S9 shows that knocking down the expression of $\alpha 4 \beta 1$ with $\beta 1$ siRNA completely blocked MMP-9 localization at the B-CLL cell surface, while CD44 expression was not disturbed. Likewise, CD44 gene silencing resulted in the complete loss of surface-associated MMP-9, while the expression of the $\alpha 4$ or $\beta 1$ subunits was not affected. Transfection with a control siRNA did not affect cell surface-bound MMP-9, which colocalized with the $\alpha 4$ and $\beta 1$ subunits but not with the CD44H isoform (Figure S9).

\section{Binding of proMMP-9 to the cell surface inhibits B-CLL cell invasion through basement membranes and transendothelial migration}

We previously showed ${ }^{6}$ and have confirmed here (Figure 7A,B) that gene silencing or Ab blocking endogenous MMP-9 inhibits B-CLL cell migration and invasion. To determine whether binding of exogenous proMMP-9 to B-CLL cells had a functional role in these processes, we incubated B-CLL cells with various concentrations of proMMP-9 and measured the migration through Matrigel or HUVECs. Interestingly, a dose-response inhibition of cell migration was observed in both systems (Figure S10A,B). This was not due to a possible toxic effect of proMMP-9 since B-CLL cell viability was not affected in these experiments (shown in Figure S10C for 2 representative samples).

We next studied whether the proMMP-9 functional effect involved binding to $\alpha 4 \beta 1$ and CD44. In the absence of exogenous proMMP-9, Ab blocking or gene silencing $\alpha 4 \beta 1$ significantly abolished B-CLL cell migration through Matrigel or HUVECs in all samples studied (Figure 7A,B), confirming previous results. ${ }^{3,6}$ In contrast, inhibiting CD44 did not affect cell migration (Figure 7A,B). Adding proMMP-9 to cells with blocked $\alpha 4 \beta 1$ function or expression resulted in inhibition of cell migration, probably due to the effect of $\alpha 4 \beta 1$ itself (Figure 7A,B). However, exogenous proMMP-9 was unable to inhibit migration on cells with blocked CD44 expression or function (Figure 7A,B). These results confirmed that proMMP-9 effect on cell migration required the interaction with its specific receptors (CD44 in this case).

To determine whether the inhibitory effect involved the enzymatic activity of MMP-9, we preincubated proMMP-9 with its specific inhibitor TIMP-1 and measured the effect of the complex on B-CLL cell migration. Efficient formation of the proMMP-9/ TIMP-1 complex was confirmed by immunoprecipitation with anti-TIMP-1 Abs and subsequent analysis by gelatin zymography (Figure 7C). Moreover, this complex bound equally well to B-CLL cells as proMMP-9 alone (Figure 7D). B-CLL cell migration through Matrigel (Figure 7E) or HUVECs (Figure 7F) was significantly inhibited in all samples analyzed by TIMP-1 (average: $76 \%$ and $79 \%$ inhibition, respectively) or proMMP-9 (average: $93 \%$ and $91 \%$ inhibition, respectively). This effect was reverted in both systems by cell incubation with the proMMP-9/TIMP-1 complex (Figure 7E,F). To confirm these results, we tested the effect of the rproMMP-9mutE, which lacks enzymatic activity but binds well to B-CLL cells (Figure 4B,C). Indeed, this mutant had no effect on cell migration, while the parental rproMMP-9fl was as effective as neutrophil-derived proMMP-9 (Figure 7E,F). As expected, the rproMMP-9 $\Delta$ HemOG variant, which does not bind to B-CLL cells, had no effect on cell migration (Figure 7E,F). These results clearly established that surface-bound MMP-9 may regulate B-CLL cell migration and this requires its proteolytic activity. 
A
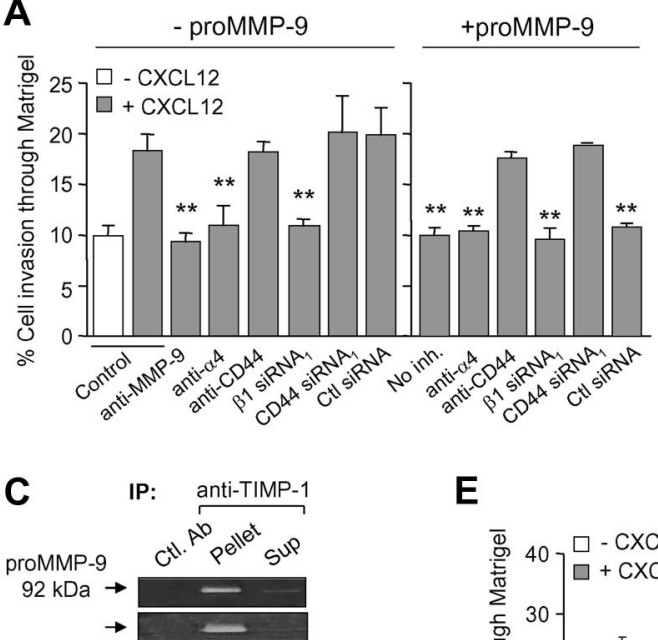

D +proMMP-9 +proMMP-9/TIMP-1

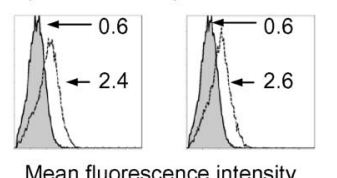

B

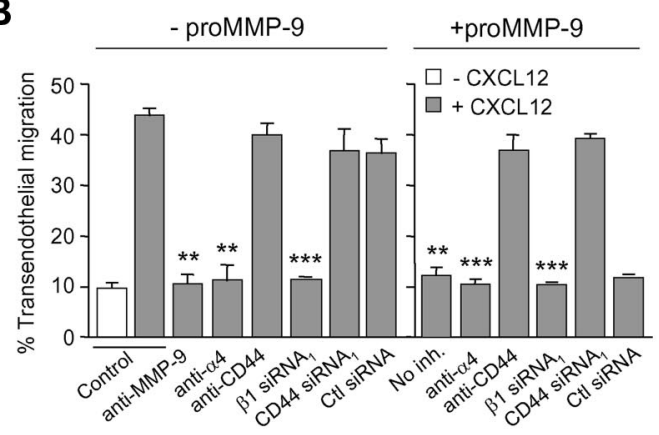

$\mathbf{F}$
$\mathbf{E}$

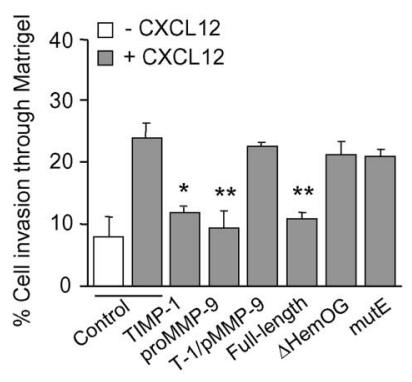

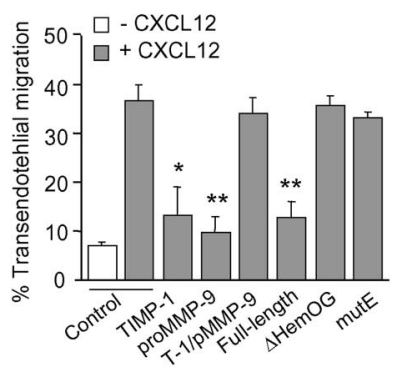

Figure 7. Functional effect of proMMP-9 binding on B-CLL cell migration through Matrigel and HUVECs. B-CLL cells $\left(5 \times 10^{5}\right)$, treated or not with the indicated Abs or transfected with the indicated siRNAs, were incubated for 30 minutes with or without $10 \mu \mathrm{g} / \mathrm{mL}$ proMMP-9. Cells were added to the upper chamber of Transwell filters coated with either Matrigel (A) or TNF- $\alpha$-activated HUVECs (B), and $150 \mathrm{ng} / \mathrm{mL} \mathrm{CXCL12} \mathrm{was} \mathrm{added} \mathrm{to} \mathrm{the} \mathrm{medium} \mathrm{in} \mathrm{the} \mathrm{bottom} \mathrm{chamber.} \mathrm{After} 24$ hours, migrated cells were counted by flow cytometry. Average values $(n=3)$ represent the percentage of the total number of cells added. (C) proMMP-9 was incubated in solution with TIMP-1 (110 nM each) for 30 minutes and the complex immunoprecipitated with anti-TIMP-1 or anti-HLA (Ctl) mAbs and protein G beads. Immunoprecipitates and supernatants were analyzed by gelatin zymography. (D) B-CLL cells from a representative sample were incubated for 30 minutes with proMMP-9 alone or complexed to TIMP-1, and analyzed by flow cytometry using anti-MMP-9 pAbs. Numbers indicate MFI values for basal MMP-9 (shaded areas) or exogenously added proMMP-9 or proMMP-9/TIMP-1 (continuous lines). $(\mathrm{E}, \mathrm{F}) \mathrm{B}-\mathrm{CLL}$ cells $\left(5 \times 10^{5}\right)$ were preincubated with or without the indicated proteins for 30 minutes at $4^{\circ} \mathrm{C}$. Cells were washed and added to Transwell filters coated with Matrigel (E) or TNF- $\alpha$-activated HUVECs $(F)$, and migration was determined after 24 hours by flow cytometry. Average values $(n=3)$ are expressed as the percentage of the total number of cells added. T-1/pMMP-9 indicates TIMP-1/proMMP-9 complex. ${ }^{\star} P \leq .05 ;{ }^{\star \star} P \leq .01$.

\section{Discussion}

We have studied the molecular associations and possible function of MMP-9 at the B-CLL cell surface. We show for the first time that (1) B-CLL cells bind soluble proMMP-9 and active MMP-9 and attach to immobilized proMMP-9 and active MMP-9; (2) these interactions are mediated by $\alpha 4 \beta 1$ integrin, $190-\mathrm{kDa} C D 44 \mathrm{v}$, and the MMP-9 hemopexin domain; (3) $\alpha 4 \beta 1$ and CD44v constitute a novel cell surface docking complex for MMP-9; (4) MMP-9 colocalizes at the cell surface with $\alpha 4 \beta 1$ and CD44v, but not with $\mathrm{CD} 44 \mathrm{H}$; and (5) surface-bound MMP-9 may regulate B-CLL cell arrest and movement.

MMP-9 has previously been shown to interact with $\alpha \mathrm{L} \beta 2$, $\alpha \mathrm{M} \beta 2, \alpha \mathrm{V} \beta 3$, and $\alpha \mathrm{V} \beta 5$ integrins on various normal and tumor cell types. ${ }^{33,34}$ We show in the present study that $\alpha 4 \beta 1$ integrin is a novel docking receptor for proMMP-9 and active MMP-9 in B-CLL cells. Since $\alpha 4 \beta 1$ up-regulates proMMP- $9,{ }^{6}$ and this MMP was shown to be involved in B-CLL cell movement, ${ }^{6,11} \alpha 4 \beta 1$ may constitute a crucial player in the progression of this malignancy. Indeed, elevated expression of $\alpha 4 \beta 1$ correlates with the presence of lymphadenopathy, ${ }^{3}$ and $\alpha 4 \beta 1$ is required for chemokine-directed B-CLL cell transendothelial migration. ${ }^{3,5}$ Moreover, the correlated overexpression of the $\alpha 4$ integrin subunit and CD38 appears to be characteristic of bad B-CLL prognosis. ${ }^{35,36}$ Our present results support these findings and establish a novel function for $\alpha 4 \beta 1$ in B-CLL (ie, anchoring MMP-9).

Our study also shows that the hyaluronan receptor CD44 ${ }^{37,38}$ is another specific receptor for proMMP-9 and active MMP-9 in
B-CLL cells. CD44 was previously shown to anchor MMP-9 at the surface of human melanoma cells, and this involved both the standard $\mathrm{CD} 44 \mathrm{H}$ form as well as some CD44v isoforms. ${ }^{15}$ In contrast with this study, proMMP-9 in B-CLL cells specifically interacted and colocalized with a 190-kDa CD44v isoform but not with $\mathrm{CD} 44 \mathrm{H}$, which is highly abundant in these cells. As mentioned for $\alpha 4 \beta 1$, overexpression of cellular CD $44 \mathrm{v},{ }^{39}$ in B-CLL or presence of this form in serum, ${ }^{40,41}$ correlates with a more aggressive disease and bad prognosis. This suggests that CD44v, like $\alpha 4 \beta 1$, may also play a role in B-CLL progression. As we show in the present study, one of the CD44v functions is to contribute to and apparently be a determinant of proMMP-9 binding to B-CLL cells. B cells lacking CD44v were unable to bind proMMP-9, albeit expressing fully functional $\alpha 4 \beta 1$ integrin. In addition, our results clearly show that $\alpha 4 \beta 1$ integrin and CD $44 v$ interact at the B-CLL cell surface, indicating that they constitute a docking complex in which both components are necessary for proMMP-9 surface binding. This complex appears to function only when cell associated as, in results not shown, we did not observe proMMP-9 binding to isolated $\mathrm{CD} 44 \mathrm{v}, \alpha 4 \beta 1$ integrin, or both proteins together, using solid-phase binding assays. One possible explanation for this could be that the docking complex contains another component(s), not yet identified, that regulates binding of proMMP-9, or serves to bridge $\alpha 4 \beta 1$, CD $44 \mathrm{v}$, and proMMP-9. Such bridging, or/and regulatory function for MMP-9 cell surface binding, has already been described for dentin ${ }^{42}$ and osteopontin ${ }^{43}$ in colon and prostate cancer cells, respectively. While these issues deserve further study, the present report is the first to show a 
specific and apparently exclusive interaction between $\alpha 4 \beta 1$ integrin and CD44v and a functional role for this complex.

Using recombinant proMMP-9 variants, we have demonstrated that proMMP-9 interaction with this docking complex involves mainly the hemopexin domain. $\alpha 4 \beta 1$ integrin and $C D 44 v$ are therefore novel ligands for this domain. In several cell types, including B-CLL cells, ${ }^{6}$ cell-associated MMP-9 is found in membrane-linked cytoskeletal superstructures for tissue invasion, ${ }^{8,9,14,34}$ suggesting that MMP-9 targeting to the cell membrane (via the hemopexin domain) may be a mechanism of controlling tumor progression. In the present report, we have studied the possible functional role of surface-bound proMMP-9 on B-CLL cell migration. Although we previously showed, ${ }^{6}$ and confirmed here, that endogenous MMP-9 appears to be required for B-CLL movement, binding of exogenous proMMP-9 to B-CLL cells clearly impaired directed cell migration in a chemotactic gradient. The proteolytic activity of MMP-9 was necessary for this effect, as established from the results with a TIMP-1/proMMP-9 complex and an inactive recombinant proMMP-9 mutant, which did not affect cell migration. As proMMP-9 represents the inactive enzyme, our findings strongly suggest that interaction of its hemopexin domain with $\alpha 4 \beta 1$ and $\mathrm{CD} 44 \mathrm{v}$ results in activation of proMMP-9 at the cell surface.

The mechanism involved in the observed functional effect is currently unknown. Given the diversity of MMP-9 targets, ${ }^{7,44}$ it is possible that when present at certain concentrations, surface-bound MMP-9 cleaves substrates that are necessary for B-CLL cell migration or/and induces cell adhesion. Opposite functional effects according to the levels of MMP-9 expression have also been observed in other tumor cell systems. ${ }^{45-48}$ It is therefore conceivable that B-CLL cells that have migrated to the bone marrow or lymph nodes increase their MMP-9 production, due to the action of chemokines. ${ }^{6}$ Indeed, immunohistochemical studies have demonstrated that B-CLL cells showing a diffused tissue infiltration pattern (bad prognosis) express high levels of MMP-9. ${ }^{11}$ MMP-9 from other sources may also be present on these tissues and facilitate B-CLL cell adhesion and arrest, rather than migration, producing the observed accumulation of malignant cells typical of B-CLL progression. The fact that the chemokine CXCL12 is a substrate of MMP-9 might also be relevant in B-CLL biology. Indeed, amino-terminal clipping of 4 residues of CXCL12 inactivates the molecule ${ }^{49}$ and might destroy the chemotactic gradient in the bone marrow. Because CXCL12 is a major chemoattractant for CXCR4-positive cells, B-CLL clones that have reached the bone marrow might become arrested and resident. The combination of the MMP-9 proteolytic activity and its ability to mediate cell arrest may therefore be crucial for B-CLL cell extravasation and localization in tissues.
In summary, the present report is the first to identify $\alpha 4 \beta 1$ integrin and CD44v as a cell surface docking molecular complex for proMMP-9 and active MMP-9. Our results can explain the observed B-CLL cell-associated proMMP-9 and provide evidence for a functional role of this MMP in the regulation of cell arrest and movement in B-CLL. Understanding the regulation and function of molecules involved in these processes is crucial for future therapies aimed to prevent B-CLL progression.

\section{Acknowledgments}

We thank the B-CLL patients who donated samples for this research; Drs Paloma Sánchez-Mateos and Rafael Samaniego (Hospital Gregorio Marañón, Madrid, Spain) for performing the confocal microscopy analyses and for valuable advice on the results; Dr Luisa Botella (Centro de Investigaciones Biológicas, Madrid, Spain) for HUVECs; Dr Ivan Stamenkovic (University of Lausanne, Lausanne, Switzerland) for the CD44 cDNAs; Dr Martin Humphries and Sue Craig (Welcome Trust Center, Manchester, United Kingdom) for the purified $\alpha 4 \beta 1$ integrin; Dr Pedro Lastres for help with flow cytometry; and Ilse Van Aelst for excellent technical assistance.

This work was supported by grants PI060400 (A.G.-P.) and PI061637 (M.J.T.) from the Ministerio de Sanidad y Consumo, and by the Fundación de Investigación Médica Mutua Madrileña (A.G.-P.). Research on the human proMMP-9 mutants was supported by the Geconcerteerde OnderzoeksActies (GOA 2007-2011) and the Fund for Scientific Research-Flanders (FWO-Vlaanderen). J.R.-M. was supported by a fellowship from the Fundación Ramón Areces. P.E.V.S. is a postdoctoral fellow of the FWO-Vlaanderen.

\section{Authorship}

Contribution: J.R.-M. performed most of the research and designed some experiments; E.U.-B. performed some experiments; J.A.G.-M. and M.J.T. contributed patient samples and clinical data; M.H.C. purified, characterized, and maintained cells; P.E.V.S. and G.O. prepared and characterized the recombinant proMMP-9 variants and reviewed the paper; A.G.-P. designed and supervised research and wrote the paper.

Conflict-of-interest disclosure: The authors declare no competing financial interests.

Correspondence: Angeles García-Pardo, Departamento de Fisiopatología Celular y Molecular, Centro de Investigaciones Biológicas, CSIC, Ramiro de Maeztu 9, 28040 Madrid, Spain; e-mail: agarciapardo@cib.csic.es.

\section{References}

1. Zent CS, Kay NE. Advances in the understanding of biology and prognosis in chronic lymphocytic leukemia. Curr Oncol Rep. 2004;6:348-354.

2. Rai KR. Pathophysiologic mechanisms of chronic lymphocytic leukemia and their application to therapy. Exp Hematol. 2007;35:134-136.

3. Till KJ, Lin K, Zuzel M, Cawley JC. The chemokine receptor CCR7 and $\alpha 4$ integrin are important for migration of chronic lymphocytic leukemia cells into lymph nodes. Blood. 2002;99:29772984.

4. López-Giral S, Quintana NE, Cabrerizo M, et al. Chemokine receptors that mediate $\mathrm{B}$ cell homing to secondary lymphoid tissues are highly expressed in B cell chronic lymphocytic leukemia and non-Hodgkin lymphomas with widespread nodular dissemination. J Leuk Biol. 2004;76:462471.

5. Till KJ, Spiller DG, Harris RJ, et al. CLL, but not normal, $B$ cells are dependent on autocrine VEGF and $\alpha 4 \beta 1$ integrin for chemokine-induced motility on and through endothelium. Blood. 2005; 105:4813-4819.

6. Redondo-Muñoz J, Escobar-Díaz E, Samaniego $\mathrm{R}$, et al. MMP-9 in B-cell chronic lymphocytic leukemia is up-regulated by $\alpha 4 \beta 1$ integrin or CXCR 4 engagement via distinct signaling pathways, localizes to podosomes, and is involved in cell invasion and migration. Blood. 2006;108:3143-3151.

7. Egeblad M, Werb Z. New functions for the matrix metalloproteinases in cancer progression. Nature Rev Cancer. 2002;2:161-176.

8. Stamenkovic I. Extracellular matrix remodelling: the role of matrix metalloproteinases. J Pathol. 2003;200:448-464.

9. Deryugina EI, Quigley JP. Matrix metalloproteinases and tumor metastasis. Cancer Metastasis Rev. 2006;25:9-34.

10. Bauvois B, Dumont J, Mathiot C, Kolb JP. Production of matrix metalloproteinase- 9 in early stage B-CLL: suppression by interferons. Leukemia. 2002;16:791-798.

11. Kamiguti AS, Lee ES, Till KJ, et al. The role of matrix metalloproteinase- 9 in the patogenesis of 
chronic lymphocytic leukemia. $\mathrm{Br} \mathrm{J}$ Haematol. 2004;125:128-140.

12. Redondo-Muñoz J, Terol MJ, García-Marco JA, García-Pardo A. Matrix metalloproteinase- 9 is up-regulated by CCL21/CCR7 interaction via extracellular signal-regulated kinase-1/2 signaling and is involved in CCL21-driven B-cell chronic lymphocytic leukemia cell invasion and migration. Blood. 2008;111:383-386.

13. Fridman R, Toth M, Chvyrkova I, et al. Cell surface association of matrix metalloproteinase- 9 (gelatinase B). Cancer Metastasis Rev. 2003;22: 153-166.

14. Bourguignon LYW, Gunja-Smith Z, lida N, et al. $\mathrm{CD}_{44} \mathrm{v}_{3,8-10}$ is involved in cytoskeleton-mediated tumor cell migration and matrix metalloproteinase (MMP-9) association in metastatic breast cancer cells. J Cell Physiol. 1998;176:206-215.

15. Yu Q, Stamenkovic I. Localization of matrix metalloproteinase 9 to the cell surface provides a mechanism for CD44-mediated tumor invasion. Genes Dev. 1999;13:35-48.

16. Stefanidakis M, Ruohtula T, Borregaard N, et al Intracellular and cell surface localization of complex between $\alpha \mathrm{M} \beta 2$ integrin and promatrix metalloproteinase- 9 progelatinase in neutrophils. J Immunol. 2004;172:7060-7068.

17. Olson MW, Toth M, Gervasi DC, et al. High affinity binding of latent matrix metalloproteinase- 9 to the alpha2(IV) chain of collagen IV. J Biol Chem. 1998;273:10672-10681.

18. Monferran S, Paupert J, Dauvillier S, et al. The membrane form of the DNA repair protein Ku interacts at the cell surface with metalloproteinase 9. EMBO J. 2004;23:3758-3768

19. Yu Q, Stamenkovic I. Cell surface-localized matrix metalloproteinase-9 proteolytically activates TGF- $\beta$ and promotes tumor invasion and angiogenesis. Genes Dev. 2000;14:163-176.

20. Oh J, Takahashi R, Kondo S, et al. The membrane-anchored MMP inhibitor RECK is a key regulador of extracellular matrix integrity and angiogenesis. Cell. 2001;107:789-800.

21. Hahn-Dantona E, Ruiz JF, Bornstein P, Strickland DK. The low density lipoprotein receptor-related protein modulates levels of matrix metalloproteinase 9 (MMP-9) by mediating its cellular catabolism. J Biol Chem. 2001;276:15498-15503.

22 Binet JL, Auqier A, Dighiero G, el al. A new prognostic classification of chronic lymphocytic leukemia derived from a multivariate analysis. Cancer. 1981;48:198-206.

23. Rai KR, Sawitsky A, Cronkite EP, et al. Clinical staging of chronic lymphocytic leukemia. Blood. 1975;46:219-234.

24. Hus I, Podhorecka M, Bojarska-Junak A, et al The clinical significance of ZAP-70 and CD38 expression in B-cell chronic lymphocytic leukaemia. Ann Oncol. 2006;17:683-690.
25. Deaglio S, Vaisitti T, Aydin S, et al. CD38 and ZAP-70 are functionally linked and mark CLL cells with high migratory potential. Blood. 2007; 110:4012-4021.

26. Pulido R, Elices MJ, Campanero MR, et al. Functional evidence for three distinct and independently inhibitable adhesion activities mediated by the human integrin VLA-4: correlation with distinct $\alpha 4$ epitopes. J Biol Chem. 1991;266:1024110245.

27. Humphries MJ, Komoriya A, Akiyama SK, et al. Identification of two distinct regions of the type III connecting segment of human plasma fibronectin that promote cell type specific adhesion. J Biol Chem. 1987;262:6886-6892.

28. Garcia-Pardo A, Wayner EA, Carter WG, Ferreira OC Jr. Human B lymphocytes define an alternative mechanism of adhesion to fibronectin. The interaction of the $\alpha 4 \beta 1$ integrin with the LHGPEILDVPST sequence of the type III connecting segment is sufficient to promote cell attachment. J Immunol. 1990;144:3361-3366.

29. Vincent AM, Cawley JC, Burthem J. Integrin function in chronic lymphocytic leukemia. Blood. 1996;87:4780-4788.

30. De la Fuente MT, Casanova B, García-Gila M, et al. Fibronectin interaction with $\alpha 4 \beta 1$ integrin prevents apoptosis in $\mathrm{B}$ cell chronic lymphocytic leukemia: correlation with $\mathrm{Bcl}-2$ and Bax. Leukemia. 1999;13:266-274

31. Van den Steen PE, Van Aelst I, Hvidberg V, et al. The hemopexin and O-glycosylated domains tune gelatinase B/MMP-9 bioavailability via inhibition and binding to cargo receptors. J Biol Chem. 2006;281:18626-18637.

32. Irving JA, Cain G, Howard M, et al. The role of alternative splicing of the adhesion molecule, CD44, in lymphoid malignancy. J Clin Pathol. 2007;51:776-780.

33. Stefanidakis M, Koivunen E. Cell-surface association between matrix metalloproteinases and integrins: role of the complexes in leukocyte migration and cancer progression. Blood. 2006;108: 1441-1450.

34. Björklund M, Koivunen E. Gelatinase-mediated migration and invasion of cancer cells. Biochim Biophys Acta. 2005;1755:37-69.

35. Pittner BT, Shanafelt TD, Kay NE, Jelinek DF CD38 expression levels in chronic lymphocytic leukemia B cells are associated with activation marker expression and differential responses to interferon stimulation. Leukemia. 2005;19:22642272.

36. Zucchetto A, Bomben R, Dal Bo M, et al. CD49d in B-cell chronic lymphocytic leukemia: correlated expression with CD38 and prognostic relevance. Leukemia. 2006;20:523-525.

37. Naor D, Vogt Sionov R, Ish-Shalom D. CD44 structure, function, and association with the ma- lignant process. Adv Cancer Res. 1997;71:241319.

38. Ponta H, Sherman L, Herrlich PA. CD44: from adhesion molecules to signalling regulators. $\mathrm{Na}$ ture Rev Mol Cell Biol. 2003;4:33-45.

39. Zarcone D, De Rossi G, Tenca C, et al. Functional and clinical relevance of CD44 variant isoform expression on B-cell chronic lymphocytic leukemia cells. Haematologica. 1998;83:1088 1098.

40. De Rossi G, Marroni P, Paganuzzi M, et al. Increased serum levels of soluble CD44 standard, but not of variants isoforms $\mathrm{v} 5$ and $\mathrm{v} 6$, in $\mathrm{B}$ cel chronic lymphocytic leukemia. Leukemia. 1997; 11:134-141.

41. Eisterer W, Bechter O, Soderberg O, et al. Elevated levels of soluble CD44 are associated with advanced disease and in vitro proliferation of neoplastic lymphocytes in B-cell chronic lymphocytic leukemia. Leukemia Res. 2004;28:1043 1051.

42. Karadag A, Fedarko NS, Fisher LW. Dentin matrix protein 1 enhances invasion potentiaal of colon cancer cells by bridging matrix metalloproteinase-9 to integrins and CD44. Cancer Res. 2005;65:11545-11552.

43. Desai B, Rogers MJ, Chellaiah MA. Mechanisms of osteopontin and CD44 as metastatic principles in prostate cancer cells. Mol Cancer. 2007;6:1-16.

44. Cauwe B, Van den Steen PE, Opdenakker G. The biochemical, biological, and pathological kaleidoscope of cell surface substrates processed by matrix metalloproteinases. Crit Rev Biochem Mol Biol. 2007; 42:113-185.

45. Deryugina El, Zijlstra A, Partidge JJ, et al. Unexpected effect of matrix metalloproteinase downregulation on vascular intravasation and metastasis of human fibrosarcoma cells selected in vivo for high rates of dissemination. Cancer Res. 2005;65:10959-10969.

46. Pozzi A, Moberg PE, Miles LA, et al. Elevated matrix metalloprotease and angiostatin levels in integrin $\alpha 1$ knockout mice cause reduced tumor vascularization. Proc Natl Acad Sci U S A. 2000; 97:2202-2207.

47. Pozzi A, LeVine WF, Gadner HA. Low plasma levels of matrix metalloproteinase 9 permit increased tumor angiogenesis. Oncogene. 2002; 21:272-281.

48. Mira E, Lacalle RA, Buesa JM, et al. Secreted MMP9 promotes angiogenesis more efficiently than constitutively active MMP9 bound to the tumor cell surface. J Cell Science. 2004;117:18471856.

49. McQuibban GA, Butler GS, Gong J-H, et al. Matrix metalloproteinase activity inactivates the CXC chemokine stromal cell-derived factor-1. J Bio Chem. 2001;276:43503-43508. 